\title{
BMJ Open Complementary feeding and the early origins of obesity risk: a study protocol
}

\author{
Naleena Devi Muniandy, ${ }^{1,2}$ Pascale A Allotey, ${ }^{1}$ Ireneous N Soyiri, ${ }^{3}$ \\ Daniel D Reidpath ${ }^{1}$
}

To cite: Muniandy ND, Allotey PA, Soyiri IN, et al. Complementary feeding and the early origins of obesity risk: a study protocol. BMJ Open 2016;6:e011635. doi:10.1136/bmjopen-2016011635

- Prepublication history for this paper is available online. To view these files please visit the journal online (http://dx.doi.org/10.1136/ bmjopen-2016-011635).

Received 22 February 2016 Revised 15 September 2016 Accepted 29 September 2016

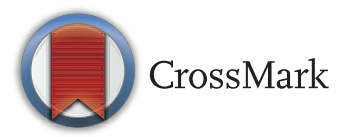

${ }^{1}$ School of Medicine and Health Sciences, Monash University Sunway Campus, Bandar Sunway, Malaysia ${ }^{2}$ Department of Nutrition and Dietetics, Faculty of Health Sciences, Mara University of Technology, Shah Alam, Malaysia

${ }^{3}$ Usher Institute for Population Health Sciences \& Informatics, Centre for Medical Informatics, The University of Edinburgh, Edinburgh, UK

Correspondence to Naleena Devi Muniandy; naleena.muniandy@monash. edu

\section{ABSTRACT}

Introduction: The rise in the prevalence of childhood obesity worldwide calls for an intervention earlier in the life cycle. Studies show that nutrition during early infancy may contribute to later obesity. Hence, this study is designed to determine if the variation in complementary feeding practices poses a risk for the development of obesity later in life. A mixed methods approach will be used in conducting this study.

Methods and analysis: The target participants are infants born from January to June 2015 in the South East Asia Community Observatory (SEACO) platform. The SEACO is a Health and Demographic Surveillance System (HDSS) that is established in the District of Segamat in the state of Johor, Malaysia. For the quantitative strand, the sociodemographic data, feeding practices, anthropometry measurement and total nutrient intake will be assessed. The assessment will occur around the time complementary feeding is expected to start (7 Months) and again at 12 months. A 24-hour diet recall and a 2-day food diary will be used to assess the food intake. For the qualitative strand, selected mothers will be interviewed to explore their infant feeding practices and factors that influence their practices and food choices in detail.

Ethics and dissemination: Ethical clearance for this study was sought through the Monash University Human Research and Ethics Committee (application number CF14/3850-2014002010). Subsequently, the findings of this study will be disseminated through peer-reviewed journals, national and international conferences.

\section{INTRODUCTION}

The theory of 'early origins of diseases', inspired by Barker et al, ${ }^{1}$ states that the environment during early development increases the susceptibility of developing obesity and other non-communicable diseases (NCDs) later in life. The exposure to an in utero environment causes permanent effects on the developing tissue. These effects are referred to as 'programming' and are important risk factors for chronic diseases in the future. ${ }^{2}$

\section{Strengths and limitations of this study}

- The study uses a mixed methods design that helps to triangulate the results from quantitative and qualitative strands to understand the research problem better.

- The study will strengthen our understanding on the influences of ecological settings on the feeding practices of a child.

- The results obtained from this study cannot be generalised to populations of other countries, but the approach may inform other research.

The window of the susceptibility period for the early origin of diseases extends from conception through infancy. Barker ${ }^{3}$ reported that the first 1000 days after conception are the most critical because most of the development takes place during this period. Recent findings highlight the potential link between health in later life and the feeding practices of the child in the first 2 years of life, including breastfeeding and complementary feeding practices. ${ }^{4}$

Nutrition intake during the prenatal stage and the first 2 years of life has been identified as an important factor contributing to future obesity. ${ }^{2-7}$ It is established that breast feeding has a protective effect over the development of obesity. ${ }^{6}{ }^{8}$ New research has begun to focus on the potential effect of complementary feeding practices on the risk of developing obesity later in life. ${ }^{9}$

Complementary feeding is defined as the process of initiating solid food and liquids when breast milk or other types of milk on their own are no longer sufficient to meet the nutritional requirements of infants. ${ }^{10}$ The WHO recommends that complementary feeding should not be introduced before 6 months, and complementary foods should supplement breast milk. The variety of foods should be introduced in amounts, frequency and consistency required to meet the nutritional needs of the infant. ${ }^{11}$

Inappropriate timing of the introduction of complementary food and an inadequate 
quantity or quality of the complementary food can lead to overnutrition or undernutrition in a child. Both these conditions during infancy increase the risk of obesity later in life. ${ }^{12}{ }^{13}$ Schwartz et al ${ }^{14}$ highlighted the importance of complementary feeding because it is a critical phase in the optimal development of behaviour in eating practices. The development of a child's eating practices is largely influenced by parental practice, environment and culture.

The strongest evidence until now linking complementary feeding and the early risk of obesity refers to timing of introduction of complementary food. ${ }^{9}{ }^{15}$ Early introduction to complementary food results in a rapid early weight gain that is associated with increased risk of obesity in later life. ${ }^{4}$ However, there are several other factors associated with complementary feeding that are not well understood. For this reason, it is worth exploring the effect of the quantity and quality of complementary food and the food preferences developed by the infant on weight gain and obesity risk. ${ }^{4916}$

Although there are links between early growth and later development of obesity, many of these findings are not consistent, and are largely influenced by a complex web of ecological determinants. ${ }^{17}$ Factors such as ethnicity, family dynamics, familial feeding practices, degree of urbanisation and mass media strongly influence complementary feeding practices of a child. ${ }^{18-22}$ However, the pathways and the interaction between these factors are not well understood.

Taking Bronfenbrenner's ecological systems framework, this study aims to investigate the variation in complementary feeding practices and the influence on the early risk factors for obesity. Determinants of early risk of obesity are (1) growth acceleration and (2) development of obesogenic food preferences. Obesogenic eating habits refer to a sustained long-term high consumption of energy dense food and liquids eventually leading to obesity. ${ }^{23} 24$

Bronfenbrenner's ecological model ${ }^{25}$ will be used in this study because it provides the framework to structure and place into context the complex influences of family history, ethnicity, socioeconomic disparities, urbanisation and mass media as factors mitigating these early risk factors for obesity.

\section{RESEARCH OBJECTIVES}

\section{Main objectives}

- To explore the variation in complementary feeding practices.

- To describe the relationship between feeding practices and early risk factors for obesity.

\section{Specific objectives}

1. To explore the ecological variation and the potential influence on complementary feeding practices.

Bronfenbrenner's ecological model ${ }^{25}$ will be used as the methodological framework in this study to explore the direct and indirect effects of the infants' setting on the variation in complementary feeding practices.

2. To describe the variation in complementary feeding practices and the total nutrition intake of the infants. The variation in complementary feeding practices in this study is operationally defined as the timing of introduction of solid foods, the types of food introduced to the infant and the macronutrient and micronutrient content of the food. The variation will also be explored against the ecological factors highlighted earlier.

3. To assess the relationship between the growth of the infants pre and post introduction of complementary feeding.

The growth measurements that will be taken are weight, length, mid upper arm circumference; head circumference and skinfold thickness.

\section{METHODS AND DESIGN}

This is a mixed methods longitudinal study designed to collect data using surveys and in-depth interviews. The quantitative section will involve a prospective cohort and will address all three of the general objectives. The qualitative section will be carried out using a grounded theory approach and is designed to answer the first and second objectives in order to be able to further explore the infant feeding practices (IFP). Preliminary analysis from the first phase of the quantitative study will inform the study population and interview guide for the qualitative study. This study design is presented in figure 1 .

A mixed methods design was chosen for this study based on previous literature which has shown that integrating quantitative and qualitative methodological approaches strengthens the overall research design and provides more comprehensive and robust evidence. Based on the six major mixed methods typologies proposed by Creswell and Clark, 2007, ${ }^{26}$ this study used the embedded design, in which the qualitative component is embedded inside the quantitative component which was designed to answer the main objective of the study.

The whole study is divided into two phases. The first phase of the study will be conducted when the infants are 7 months old. This age is chosen because complementary food is supposed to be introduced after 6 months. ${ }^{11}$ Meeting the participants at 7 months allows us to capture the early practices immediately after the likely introduction of complementary food. The second phase of the study will be conducted when the infants are 12 months old. This age is chosen as infants would have been introduced to different types and textures of food $^{27}$ and have also developed food preference by this time. ${ }^{28}$ Since studies have shown that weight gain during infancy may predict later obesity, ${ }^{29}$ this study allows us to detect whether complementary feeding practices of these infants result in a divergence from the recommended growth. In addition, selected mothers of the 


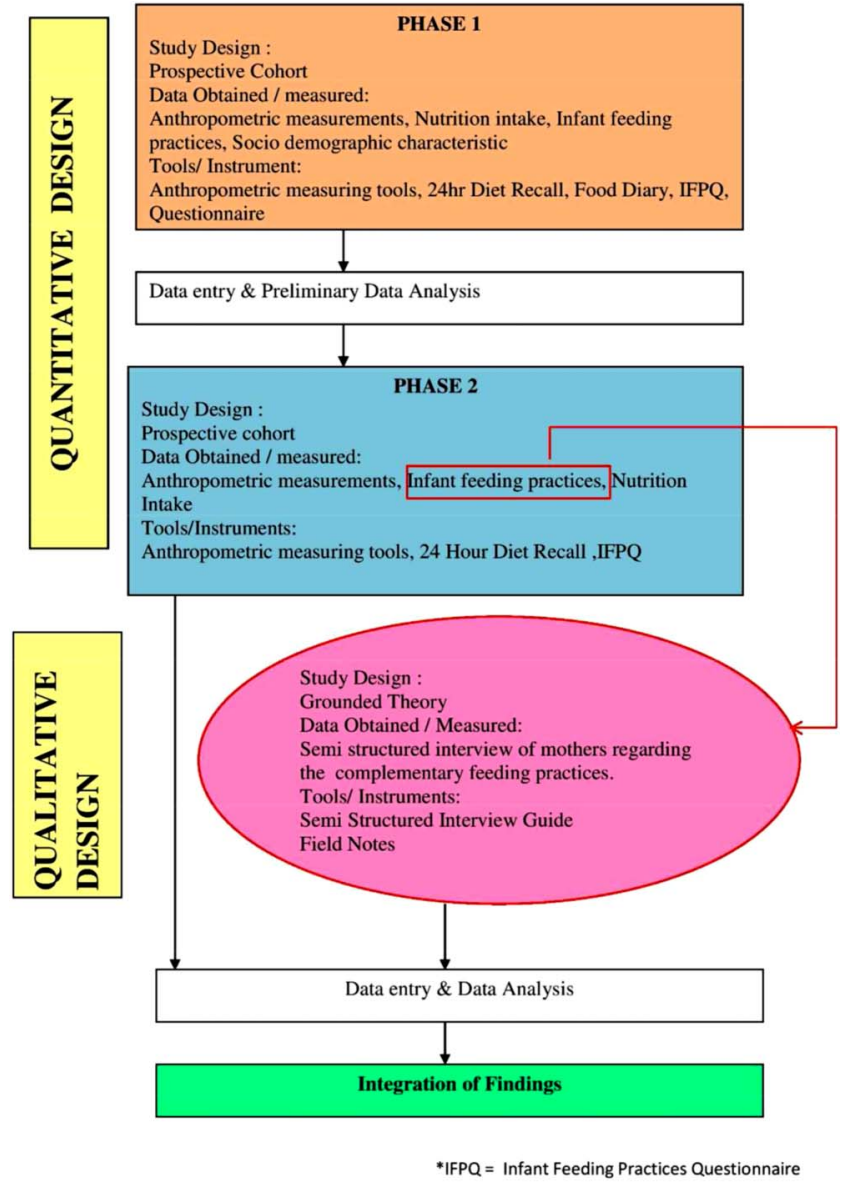

Figure 1 Mixed methods embedded design for the study of complementary feeding and the early origins of obesity risk.

infants will be interviewed using an in-depth interview method during this second phase to explore food choices and complementary feeding practices in greater detail.

\section{Study location}

This study will be conducted in the South East Asia Community Observatory (SEACO) research platform. SEACO is a Health and Demographic Surveillance System (HDSS), established in the District of Segamat, in the state of Johor, Malaysia. ${ }^{30}$ SEACO covers 5 of the 11 subdistricts (Mukim) in the District of Segamat. This platform collects longitudinal data on the entire population within the five districts. The five subdistricts under SEACO are Bekok, Jabi, Sungai Segamat, Gemereh and Chaah.

Agriculture is the main contributor to the economy in all the subdistricts in Segamat, including the ones in SEACO, followed by the manufacturing and government service sectors. According to the national census (2010), the total population in Segamat is 170000 of which 50\% are Malays and Orang Asli, 36\% are Chinese and 9\% are Indians. This ethnic composition is very similar to the actual population breakdown of the country. ${ }^{31}$ The multiethnic setting in SEACO provides the context to understand how the ecological factors influence complementary feeding practices.

\section{Study population}

Mothers of infants born between the month of January to June 2015 will be identified from the SEACO demographic database and approached. These infants, together with their mothers, will be recruited in this study if they meet the inclusion and exclusion criteria.

Inclusion criteria:

1. Singleton pregnancy.

2. Full-term infants: $37-42$ gestation weeks.

3. Birth weight of the infant should be between 2.5 and $4.0 \mathrm{~kg}$.

Infants weighing $<2500 \mathrm{~g}$ (small for gestation age, SGA), $>4000 \mathrm{~g}$ (large for gestational age, LGA) and infants born before 37 weeks of gestation will be excluded from this study. Studies have shown that both SGA and LGA babies may develop metabolic syndrome later in life. ${ }^{32}$ SGA and infants born before 37 weeks of gestation have a higher risk of early growth retardation, infection, development delay and need special care. ${ }^{33}$

Exclusion criteria:

1. Infants with a medical condition at birth that would affect feeding practices or the appetite of the child.

2. Infants who have been admitted to an intensive care unit for 3 or more days.

3. Infants whose mothers are aged below 18 years.

4. Infants who are not of Malay, Chinese, Indian or 'Orang Asli' ethnicity.

Infants with a medical condition such as cerebral palsy, neurological disorders or congenital effects are excluded from this study. These conditions may impair their nutritional intake and feeding practices. ${ }^{34} 35$ Infants born to teenage mothers are excluded from this study because teenage pregnancy is associated with a higher risk of neonatal medical complications. ${ }^{36}$ Also, infants who are not from the four ethnic groups stated will not be included in this study as the total number of participants might be too few to show any statistical significance.

\section{Quantitative strand}

Sample size

All infants born within the SEACO surveillance site between 1 January 2015 and 30 June 2015 will be recruited in this study. Around 200 mother-infants pairs have been identified from the SEACO database. These infants will be approached for the first phase of the study at 7 months of age and again when they are 12 months old, for the second phase of the study. The sample size was determined on the basis of feasibility rather than a power calculation of an essentially arbitrary and fixed hypothesis test.

\section{Data collection}

The first step is to identify the target participants from the SEACO database. Mothers of the infants will be 
contacted via telephone to identify if the infant and mother still reside in the given address. Before proceeding with the data collection, consent will be obtained from the mothers as part of the recruitment process.

Data collectors will be recruited and trained to assist in data collection and follow-up. The data collectors recruited for this study need to be fluent in Malay and English. They will be trained to obtain anthropometry measurements and diet history from the participants. An interpreter may be recruited to assist in interviewing participants who are unable to communicate in Malay or English.

The first phase of data collections will be conducted when the infants are at least 7 months old. At this stage, the data collectors will take the anthropometry measurements of the infant. The infant's weight, length, head circumference, mid-arm circumference and skinfold thickness will be taken. For the mother, the prepregnancy weight and weight gain will be obtained from their 'Prenatal Record Book'. For those who have lost the book, the verbal information from the mothers will be collected. A questionnaire will be used to record the sociodemographic particulars and IFP. The IFP survey will be part of the main questionnaire and will be used to obtain the infant's breastfeeding, bottle-feeding and complementary feeding practices.

During this stage too, infants' particulars such as birth weight, gestational age and medical history will be taken. This information can be obtained from the 'Baby Record Book'. Maternal medical history and complications during pregnancy will also be obtained. After this session, information obtained will be keyed in a statistical analysis package.

The second phase of data collection will be conducted when the infants are 12 months old. During this phase, the anthropometric measurements of the infants will be taken again. The feeding practices will be assessed using an IFP survey. The total nutrient intake of the infant will be assessed using a 24-hour diet recall (1 day) and a food diary (2 days).

At the end of the session, the data will be organised using the statistical analysis package, $\mathrm{R}$ while the 24-hour diet recall and the 2 days food diary will be analysed using the nutrition analysis package, Nutritionist Pro. The anthropometry measurements of the infants will be compared with the standard growth curves using the WHO growth charts. ${ }^{37}$ The macronutrient and micronutrient intake of the child will be compared with the national recommendations using the Recommended Nutrition Intake of Malaysia. ${ }^{38}$

The flow chart of the data collection process is presented in figure 2.

\section{Measurement instruments and outcomes measures Anthropometry measurements}

The infant's anthropometric measurements that will be taken include head circumference, mid-upper arm circumference, weight, length and skinfold thickness. In addition, maternal weight will also be measured in both the phases. Body weight will be recorded in kilogram, while height, head circumference and mid-upper arm circumferences will be recorded in centimetre and skinfold thickness will be recorded in millimetre. All the measurements will be taken by referring to the National Health and Nutrition Examination Survey (NHANES) Anthropometry Procedure Manual (2007). ${ }^{39}$ In this study, all the measurements will be taken and recorded by trained data collectors.

Infant's birth weight, gestation age at delivery and any medical history from birth until the present day will be captured in the questionnaire. In addition, feeding difficulties, history of hospitalisation and information on other siblings of the infant will also be obtained.

\section{Dietary intake}

The dietary intake of the infants will be collected using an Infant Feeding Style Survey (IFSS). This survey will be embedded in the main questionnaire and will be used in both stages of the data collection. This part of the study will capture the diet transition of the infant from birth until 12 months. Detailed questions regarding prelacteal feeds, breastfeeding practices, bottle-feeding practices and complementary feeding practices will be obtained here.

The IFSS was adapted from the Infant Feeding Style Questionnaire used in the Feeding Infants and Toddlers Study (FITS $)^{40} 41$ and from the multiple questionnaires that were used in the Infant Feeding Practices Study II. ${ }^{42}$ Minor adaptation was made to suit the type of food available locally.

Assessing dietary intake in people of any age is a challenging process. However, measuring the diet intake in infants and toddlers is even more difficult because we can only obtain their diet recall information through their parents or caregivers. This is complicated because multiple people may be responsible for a child's diet intake in a day. ${ }^{43}$ Trolle $e t a t^{44}$ have suggested that using a 24-hour diet recall in combination with a food record book will provide a more accurate estimation of a child's diet intake, compared with using just one method in obtaining the infant's diet intake.

In this study, a 24-hour diet recall of the infant will be obtained from the mothers. In addition, a food diary will be provided to the mothers to fill in their child's diet intake for 2 days. Pictures of food and household measurements will be provided in the food diary in order to assist the mothers to fill in the diet intake of the child more accurately.

\section{Sociodemographic and other relevant data}

A questionnaire will be used to obtain sociodemographic information, as well as the infant's medical and clinical history and maternal medical and clinical history. In the demographic section, household income, maternal age, education and employment status, pre-pregnancy weight and weight gain during pregnancy, nature of delivery, prenatal and postnatal medical history and other relevant information of the mother will be taken. 
Figure 2 Flow chart of data collection. SEACO, South East Asia Community Observatory.
Flow Chart of Data Collection

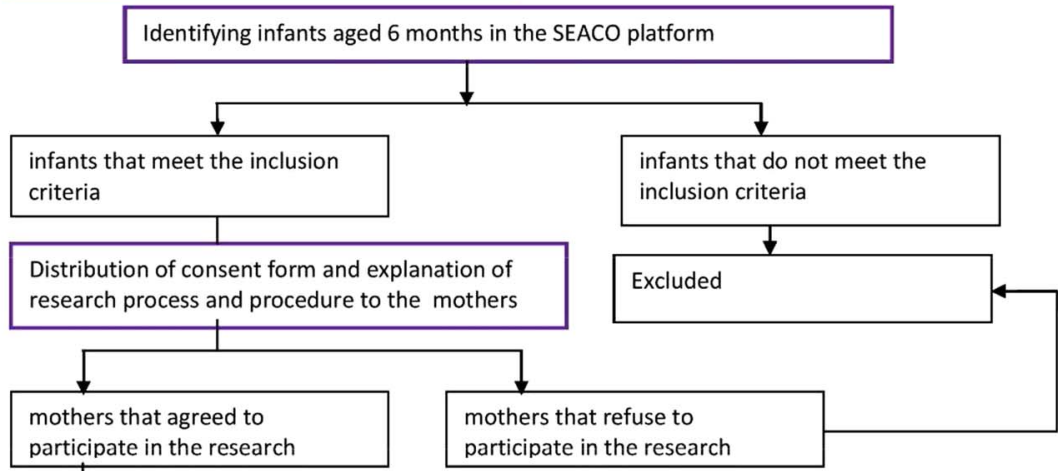

Distribution of questionnaire

- Socio demography information will be taken using a questionnaire.

- Anthropometry measurements of infants will be taken

- Feeding practices will be obtained using a Infant Feeding Practice questionnaire

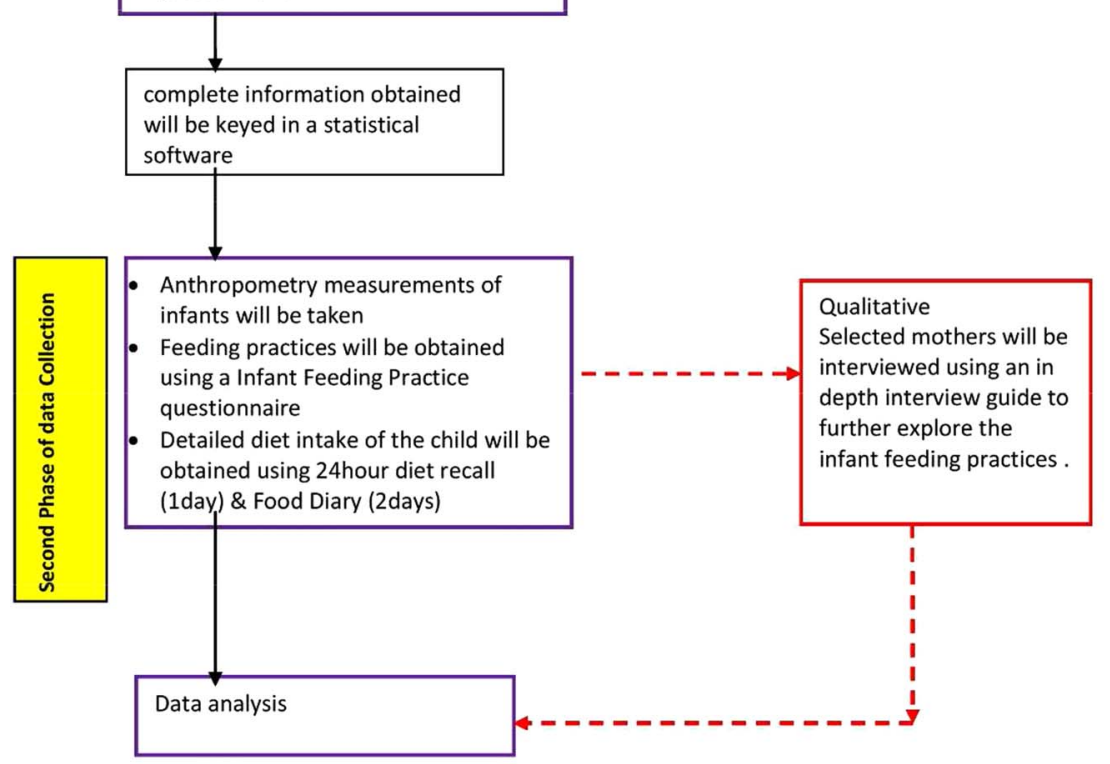

The outcome measures and measurement instruments are summarised in table 1.

\section{Statistical analysis}

Demographic data, IFP and anthropometric measurements will be entered into a statistical analysis package, 'R'. Normality checks will be performed on all continuous data prior to further analysis. Descriptive statistics will be used to report the basic sociodemographic data and the mean dietary intake of the infants. Paired Student's t-test will be used to compare the anthropometric measurement after the introduction of complementary food. Regression methods will be used to measure the relationship between complementary feeding and all the sociodemographic factors.
Qualitative strand

Sample size

A grounded theory approach will be used in the qualitative section of this study. A purposive sampling strategy will be used to identify $\sim 20$ mothers to participate in an in-depth interview. The sample size, 20 , is planned as studies have shown that a sample size smaller than 20 will be sufficient to facilitate a researcher to obtain the information needed in an in-depth interview. ${ }^{45}$ However, since theoretical models are constantly revised, more participants may be interviewed if necessary.

\section{Data collection}

For the qualitative part of this study, in-depth interviews will be carried out in the second phase of data 
Table 1 The study outcome measures and measurement instruments

\begin{tabular}{|c|c|c|}
\hline Outcome measured & Instrument/tool & Indicator \\
\hline \multicolumn{3}{|l|}{ Anthropometry } \\
\hline Infant's weight & Infant Seca electronic digital scale & Current nutritional status \\
\hline Infant's length & 'Infantometer' & Long-term nutritional status \\
\hline Infant's head circumference & Non-stretchable measuring tape & $\begin{array}{l}\text { Detect abnormalities of head and brain } \\
\text { growth }\end{array}$ \\
\hline Infant's mid-upper arm circumference & Non-stretchable measuring tape & Indicator for protein-energy malnutrition \\
\hline Infant's skinfold thickness & Skin callipers & Estimating body fat \\
\hline \multicolumn{3}{|l|}{ Diet intake } \\
\hline Macronutrient and micronutrient intake & $\begin{array}{l}24 \text {-hour diet recall ( } 2 \text { weekdays, } \\
1 \text { weekend) }\end{array}$ & Current diet intake \\
\hline Feeding practices & $\begin{array}{l}\text { Infant Feeding Practice } \\
\text { Questionnaire }\end{array}$ & Feeding practices, food preferences \\
\hline \multicolumn{3}{|l|}{ Sociodemographic/ecology determinants } \\
\hline $\begin{array}{l}\text { Family dynamics, household income, } \\
\text { ethnicity }\end{array}$ & Questionnaire & Influence on feeding practices \\
\hline
\end{tabular}

collection. Selected participants, who are mothers of the infants, will be recruited based on the nutrition status of the infants or sociodemographic status of the mothers. The participants will be interviewed on their feeding practices and factors that influence choices of food that is fed to their infants. They will be probed to describe and explain their experience in introducing complementary food to their infants. The interviews will be recorded and transcribed later.

\section{Data analysis}

Interviews, observations and 24-hour diet recalls will be analysed. The audio recorded interviews will be transcribed verbatim. The transcribed interview will be analysed using 'NVIVO' software for qualitative research. Ideas or phrases that emerge from the transcribed interviews will be organised using codes. These codes will be organised into themes and the results will be used to explain the variations in the complementary feeding practices.

\section{DISCUSSION}

The prevalence of childhood obesity is rising and multiple studies indicate that most of the risk factors develop during the early phases of life. These factors may range from the time of preconception, to the prenatal and postnatal period. Given this, this study will look into how the complementary feeding practices may influence the development of early obesity risk. In this study, it is impossible to measure the development of obesity as the infants will only be followed for a period of 5 months after the introduction to complementary food. However, this study will be one of the first to measure the early risk factors for developing obesity through the assessment of growth acceleration, predisposition of body fat and the development of obesogenic eating habits. The study is also unique in its use of the mixed methods approach that helps to triangulate the results from the quantitative and qualitative methods to understand the research problem better. In addition, this study is designed to strengthen our understanding on the influence of ecological settings on the feeding practices of a child.

Contributors NDM developed the original research design, and refined it with PAA, INS and DDR. NDM wrote the initial draft and all authors subsequently contributed to and commented on the manuscript and approved the final version.

Competing interests None declared.

Ethics approval Ethical clearance for this study was sought through the Monash University Human Research and Ethics Committee (application number CF14/3850-2014002010).

Provenance and peer review Not commissioned; externally peer reviewed.

Open Access This is an Open Access article distributed in accordance with the Creative Commons Attribution Non Commercial (CC BY-NC 4.0) license, which permits others to distribute, remix, adapt, build upon this work noncommercially, and license their derivative works on different terms, provided the original work is properly cited and the use is non-commercial. See: http:// creativecommons.org/licenses/by-nc/4.0/

\section{REFERENCES}

1. Barker D, Osmond C, Golding J, et al. Growth in utero, blood pressure in childhood and adult life, and mortality from cardiovascular disease. BMJ 1989;298:564.

2. Langley-Evans SC. Nutrition in early life and the programming of adult disease: a review. J Hum Nutr Diet 2015;28(Suppl 1):1-14.

3. Barker DJP. Developmental origins of chronic disease. Public Health 2012;126:185-9.

4. Adair LS. How could complementary feeding patterns affect the susceptibility to NCD later in life? Nutr Metab Cardiovasc Dis 2012;22:765-9.

5. Barouki R, Gluckman PD, Grandjean P, et al. Developmental origins of non-communicable disease: implications for research and public health. Environ Health 2012;11:42.

6. Young BE, Johnson SL, Krebs NF. Biological determinants linking infant weight gain and child obesity: current knowledge and future directions. Adv Nutr Int Rev J 2012;3:675-86.

7. Gillman MW. Early infancy as a critical period for development of obesity and related conditions. In: Lucas A, Makrides M, Ziegler EE, eds. Nestlé Nutrition Institute Workshop series: pediatric program. Basel: KARGER, 2010:13-24.

8. Koletzko $B$, von Kries R, Closa $R$, et al. Can infant feeding choices modulate later obesity risk? Am J Clin Nutr 2009;89:1502S-8S. 
9. Grote V, Theurich M, Koletzko B. Do complementary feeding practices predict the later risk of obesity? Curr Opin Clin Nutr Metab Care 2012;15:293-7.

10. WHO. Complementary feeding for breastfed children. http:// whqlibdoc.who.int/hq/2000/WHO_NHD_00.1.pdf (accessed 29 May 2014)

11. WHO. Complementary feeding. WHO. http://www.who.int/nutrition/ topics/complementary_feeding/en/ (accessed 12 Jun 2014).

12. Michaelsen KF, Larnkjær A, Mølgaard C. Amount and quality of dietary proteins during the first two years of life in relation to NCD risk in adulthood. Nutr Metab Cardiovasc Dis 2012;22:781-6.

13. Kuriyan R, Kurpad AV. Complementary feeding patterns in India. Nutr Metab Cardiovasc Dis 2012;22:799-805.

14. Schwartz C, Scholtens PAMJ, Lalanne A, et al. Development of healthy eating habits early in life. Review of recent evidence and selected guidelines. Appetite 2011;57:796-807.

15. Thompson AL, Bentley ME. The critical period of infant feeding for the development of early disparities in obesity. Soc Sci Med 2013;97:288-96.

16. Robinson S, Fall C. Infant nutrition and later health: a review of current evidence. Nutrients 2012;4:859-74.

17. Monasta L, Batty GD, Cattaneo A, et al. Early-life determinants of overweight and obesity: a review of systematic reviews. Obes Rev 2010;11:695-708.

18. Taveras EM, Gillman MW, Kleinman $\mathrm{K}$, et al. Racial/ethnic differences in early life risk factors for childhood obesity. Pediatrics 2010;125:686-95.

19. Enneman A, Hernández L, Campos R, et al. Dietary characteristics of complementary foods offered to Guatemalan infants vary between urban and rural settings. Nutr Res 2009;29:470-9.

20. Caroli M, Mele RM, Tomaselli MA, et al. Complementary feeding patterns in Europe with a special focus on Italy. Nutr Metab Cardiovasc Dis 2012;22:813-18.

21. Lutter CK. Growth and complementary feeding in the Americas. Nutr Metab Cardiovasc Dis 2012;22:806-12.

22. Grote V, Theurich M. Complementary feeding and obesity risk. Curr Opin Clin Nutr Metab Care 2014;17:273-7.

23. Rodgers RF, Paxton SJ, Massey R, et al. Maternal feeding practices predict weight gain and obesogenic eating behaviors in young children: a prospective study. Int J Behav Nutr Phys Act 2013;10:24.

24. Campbell K, Hesketh K, Crawford D, et al. The Infant Feeding Activity and Nutrition Trial (INFANT) an early intervention to prevent childhood obesity: cluster-randomised controlled trial. BMC Public Health 2008;8:103.

25. Bronfenbrenner U. The ecology of human development. Experiments by nature and design. Harvard University Press, 1979.

26. Creswell JW, Clark VLP. Designing and conducting mixed methods research. 2nd edn. SAGE.

27. Grummer-Strawn LM, Scanlon KS, Fein SB. Infant feeding and feeding transitions during the first year of life. Pediatrics 2008;122: S36-42.
28. Maier-Nöth A, Schaal B, Leathwood $\mathrm{P}$, et al. The lasting influences of early food-related variety experience: a longitudinal study of vegetable acceptance from 5 months to 6 years in two populations. PLOS ONE 2016:11:e0151356.

29. Druet C, Stettler N, Sharp S, et al. Prediction of childhood obesity by infancy weight gain: an individual-level meta-analysis. Paediatr Perinat Epidemiol 2012;26:19-26.

30. Allotey P, Reidpath DD, Devarajan N, et al. Cohorts and community: a case study of community engagement in the establishment of a health and demographic surveillance site in Malaysia. Glob Health Action 2014;7:23176.

31. Department of Statistics Malaysia Official Portal. https://www. statistics.gov. $\mathrm{my} /$ index.php? $\mathrm{r}=$ column/cthemeByCat\&cat $=117 \& \mathrm{bul}$ id=MDMxdHZjWTk1SjFzTzNkRXYzcVZjdz09\&menu_id=LOphe U43NWJwRWVSZkIWdzQ4TIhUUT09 (accessed 8 Oct 2015).

32. Ornoy A. Prenatal origin of obesity and their complications: gestational diabetes, maternal overweight and the paradoxical effects of fetal growth restriction and macrosomia. Reprod Toxicol 2011;32:205-12.

33. WHO. Infant and young child feeding: model chapter. WHO.

34. Prasse JE, Kikano GE. An overview of pediatric dysphagia. Clin Pediatr (Phila) 2009;48:247-51.

35. Evans S, Alroqaiba N, Daly A, et al. Feeding difficulties in children with inherited metabolic disorders: a pilot study. J Hum Nutr Diet 2012;25:209-16.

36. Ruedinger E, Cox JE. Adolescent childbearing: consequences and interventions. Curr Opin Pediatr 2012;24:446-52.

37. World Health Organization. WHO child growth standards length/ height-for-age, weight-for-age, weight-for-length, weight-for-height and body mass index-for-age: methods and development. Geneva: World Health Organization, 2006.

38. Recommended Nutrient Intakes for Malaysia. http://www.moh.gov. my/images/gallery/rni/insert.pdf (accessed 2 Nov 2014)

39. Anthropometry Procedures Manual—bodymeasures 09.pdf. http:// www.cdc.gov/nchs/data/nhanes/nhanes_09_10/bodymeasures_09. pdf (accessed 9 Sep 2014).

40. Ziegler P, Briefel R, Clusen N, et al. Feeding Infants and Toddlers Study (FITS): development of the FITS survey in comparison to other dietary survey methods. J Am Diet Assoc 2006;106(Suppl 1):S12-27.

41. Mennella JA, Ziegler P, Briefel R, et al. Feeding infants and toddlers study: the types of foods fed to Hispanic infants and toddlers. J Am Diet Assoc 2006;106:96-106.

42. Infant Feeding Practices Study II: Questionnaires I DNPAO I CDC. $\mathrm{http}: / / w w w . c d c . g o v /$ ifps/questionnaires.htm (accessed 22 May 2014).

43. Foster $\mathrm{E}$, Adamson $\mathrm{A}$. Challenges involved in measuring intake in early life: focus on methods. Proc Nutr Soc 2014;73:201-9.

44. Trolle E, Amiano P, Ege M, et al. Feasibility of repeated 24-h dietary recalls combined with a food-recording booklet, using EPIC-Soft, among preschoolers. Eur J Clin Nutr 2011;65(Suppl 1):S84-6.

45. Crouch M, McKenzie $\mathrm{H}$. The logic of small samples in interview-based qualitative research. Soc Sci Inf 2006;45:483-99. 